\title{
Optimal Design of Ferromagnetic Pole Pieces for Transmission Torque Ripple Reduction in a Magnetic-Geared Machine
}

\author{
Sung-Jin Kim*, Eui-Jong Park* and Yong-Jae Kim ${ }^{\dagger}$
}

\begin{abstract}
This paper derives an effective shape of the ferromagnetic pole pieces (low-speed rotor) for the reduction of transmission torque ripple in a magnetic-geared machine based on a Box-Behnken design (BBD). In particular, using a non-linear finite element method (FEM) based on 2-D numerical analysis, we conduct a numerical investigation and analysis between independent variables (selected by the $\mathrm{BBD}$ ) and reaction variables. In addition, we derive a regression equation for reaction variables according to the independent variables by using multiple regression analysis and analysis of variance (ANOVA). We assess the validity of the optimized design by comparing characteristics of the optimized model derived from a response surface analysis and an initial model.
\end{abstract}

Keywords: Magnetic-geared machine(MGM), Transmission torque ripple, Box-Behnken design (BBD), Finite element method(FEM), Analysis of variance(ANOVA), Response surface methodology (RSM)

\section{Introduction}

The magnetic gear $(\mathrm{MG})$ is a non-contact machine for torque transmission, acceleration, and deceleration. MGs have advantages such as no mechanical loss and maintenance-free operation [1-6]. Furthermore, MGs have inherent protection characteristics. A variety of MGs have been proposed. On the other hand, a novel structure for a magnetic-geared machine (MGM) was proposed by Atallah et al [1]. This MGM has a high- and low-speed rotor, and a stator. The stator uses coils instead of permanent magnets (PMs), and the low-speed rotor is sandwiched between the high-speed rotor and the stator. The high-speed rotor is driven by the electromotive force generated by the coils, and the low-speed rotor rotates in accordance with the gear ratio [4]. However, these MGMs exhibit high transmission torque ripple, which leads to noise and vibrations in the machine. Therefore, this paper proposes an effective shape of the ferromagnetic pole pieces (low-speed rotor) to reduce the transmission torque ripple of the MGM. The shape is based on a Box-Behnken design (BBD). Using a non-linear finite element method (FEM) based on 2-D numerical analysis, an analysis is conducted between independent variables (selected by the BBD) and reaction variables. In addition, a regression equation of the reaction variable according to the independent variable, using multiple regression analysis and analysis of variance (ANOVA), is derived. The validity of the optimized design is assessed by comparing the characteristics of an

$\uparrow$ Corresponding Author: Dept. of Electrical Engineering, Chosun University, Korea. (kimyj21@chosun.ac.kr)

* Dept. of Electrical Engineering, Chosun University, Korea (\{kimsj641,puj1988\}@chosun.kr)

Received: June 25, 2015; Accepted: April 27, 2016 optimized model derived from response surface analysis with those of an initial model.

\section{Structure and Operational Principle of the Machine}

\subsection{Analysis model}

Fig. 1 shows the MGM in this paper. The high-speed rotor has a yoke made of laminated silicon steel and 8 pole surface-mounted-type $\mathrm{PMs}$ with $\mathrm{Br}=1.28 \mathrm{~T}$. The 12-slot stator has three-phase concentrated windings. The diameter of the coil is $1.2 \mathrm{~mm}$, and the number of turns is 17 . Between the high-speed rotor and the stator, 20 modulating ferromagnetic pole pieces made of laminated silicon steel are added. These pole pieces serve as the low-speed rotor and are used to modulate the space harmonics of the airgap flux density.

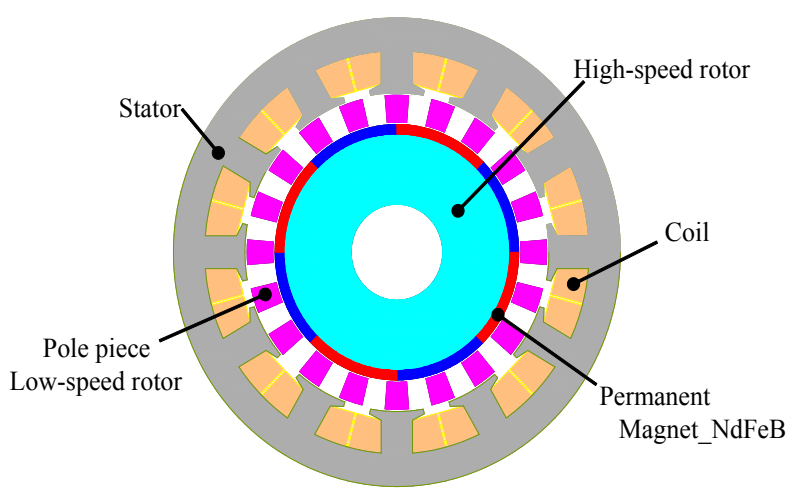

Fig. 1. Magnetic-geared machine 


\subsection{Operational principle and gear ratio}

The high-speed rotor is driven by the magnetomotive force due to the coil windings using the operational principle of conventional brushless motors. The low-speed rotor is rotated by employing the operational principle of an $\mathrm{MG}$.

The torque transmission of the MGM is based on the modulation of a magnetic field using pole pieces between the stator and high-speed rotor. As shown in (1), the number of slots in the stator is $N_{S}$, the number of magnetic pole pairs of the high-speed rotor is $N_{h}$, and the number of pole pieces of the low-speed rotor is $N_{l}$ [3-6].

$$
(2 p-1) N_{S}=(2 n-1) N_{l} \pm 2(2 m-1) N_{h}
$$

where $m, n$, and $p$ are positive integers. In addition, the gear ratio $G_{r}$ of the MGM is defined by

$$
G_{r}=\mp \frac{(2 n-1) N_{l}}{2(2 m-1) N_{h}} .
$$

In this paper, $N_{h}=4, N_{l}=20, N_{S}=12, m=n=p=1$ are selected. The gear ratio $G_{r}$ is 2.5 by (2).

\section{Reduction of Transmission Torque Ripple of MGM}

\subsection{Design of the high-speed rotor}

The design of high-speed rotor was carried out before optimal design of the ferromagnetic pole pieces for transmission torque ripple reduction. The design of highspeed rotor was conducted without the ferromagnetic pole pieces. The torque of MGM is transmitted through the ferromagnetic pole pieces. Therefore, high-speed rotor design is more advantageous than the stator design for transmission torque ripple reduction. The design parameters of the high-speed rotor are the pole arc angle and thickness of PMs. The transmission torque ripple can be reduced by

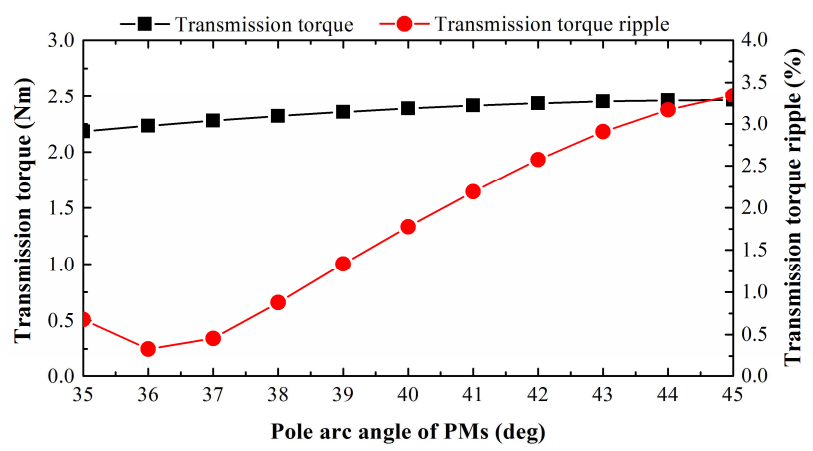

Fig. 2. Rated torque and torque ripple according to the pole arc angle of the PMs

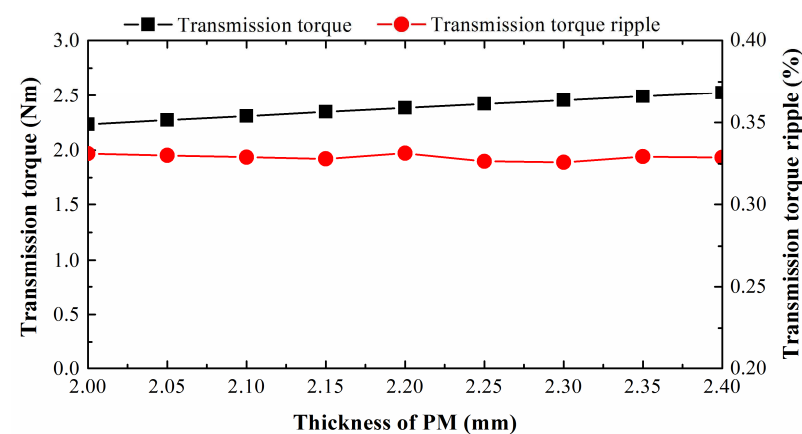

Fig. 3. Rated torque and torque ripple according to the thickness of PMs

designing pole arc angle of the PMs, but by reducing the amount of the PMs which results in a reduced output. Therefore, pole arc angle of the PMs minimizing the transmission torque ripple was designed, and thickness of PMs was adjusted to satisfy the output power. Fig. 2 shows the rated torque and torque ripple according to the pole arc angle of the PMs. As shown in Fig. 2, it can be seen that the torque ripple was reduced the most when the pole arc angle of the PMs is $36^{\circ}$. However, rated torque was decreased to $0.28 \mathrm{Nm}$ than when pole arc angle of the PMs is $45^{\circ}$. On this, thickness of PMs was designed to satisfy the output power. Fig. 3 shows the rated torque and torque ripple according to the thickness of PMs. As a result, 2.35 $\mathrm{mm}$ thickness of PMs, up from $0.35 \mathrm{~mm}$ initial model, satisfies the objective output power. After the insertion of the ferromagnetic pole pieces into derived model, analysis was proceeded to design the optimal ferromagnetic pole pieces.

Thus, transmission torque ripple is $65.81 \%$ and it is confirmed that the transmission torque ripple is increased considerably by ferromagnetic pole pieces. Therefore, optimal design of the ferromagnetic pole pieces to reduce the transmission torque ripple is proceeded in the next section.

\subsection{Response surface methodology}

Response surface methodology (RSM) is one of designs of experiment (DOE). The optimal condition of the RSM is determined through an ANOVA or the DOE. Then, an analysis is conducted of the interaction formula for input variables $x_{1}, \cdots x_{n}$ and output value $y$. In other words, the RSM is used to find the optimal response condition based on several factors. If there is a radius of curvature among the response variables, a response surface design is used to identify the relationship between two or more factors. When an unknown function between the design variables $x_{1}, \cdots x_{n}$ and the dependent variable $y$ is expressed as $f(x)$. This unknown function is called a response function. When the number of design variables $x$ is $k$ and the response function $y$ is assumed to be secondary regression model, $y$ can be expressed as 


$$
y=\beta_{0}+\sum_{i=1}^{k} \beta_{i} x_{i}+\sum_{i=1}^{k} \beta_{i i} x_{i i}{ }^{2}+\sum_{i \leq j}^{k} \beta_{i j} x_{i} x_{j}+\varepsilon
$$

where $\varepsilon$ represents a statistical error, which is generally assumed to be the normal distribution with a mean of zero and variance of $\sigma^{2}$ [7-14]. Therefore, the response function estimated from the approximation function is expressed in vector form as

$$
y=X E+\varepsilon
$$

where $X$ is the matrix of the design variables, $E$ is the regression coefficient vector, and $\varepsilon$ is the random error vector. In addition, the regression coefficient vector is estimated using the least-squares method that takes the square sum of random errors as the minimum. The leastsquare estimator of the regression coefficient vector is [714]

$$
E=\left(X^{\prime} X\right)^{-1} X^{\prime} y
$$

\subsection{Box-behnken design}

The RSM is divided into a central composite design (CCD) and the BBD used in this study. As shown in Fig. 4, each experiment point of a design with three factors can be illustrated. The BBD is used to efficiently estimate the first and second terms of the response-surface-estimate equation, when it is certain that factors are not all low level or high level at the same time, or when it is certain that all experiments are conducted in a stable region. The number of experiments that use the BBD is relatively smaller than that of the CCD. BBD is useful when experiment costs are considerably expensive or practically impossible to conduct experiments at the factorial point.

On the other hand, CCD allows for an experiment that uses a factorial point and an axial point, while BBD allows for an experiment using the center point of the factorial point. When CCD is used, a design at the axial point is needed, as shown in Fig. 4(a).

For a design at the axial point, when there are three

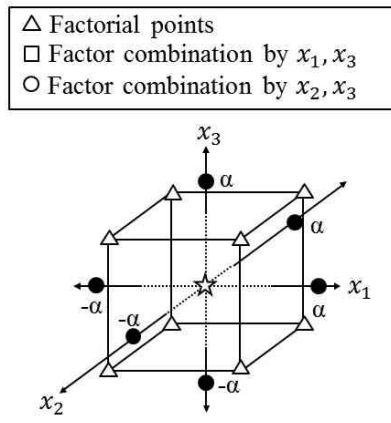

(a)

\section{$\Delta$ Factor combination by $x_{1}, x_{2}$ Center Points - Axial Points}

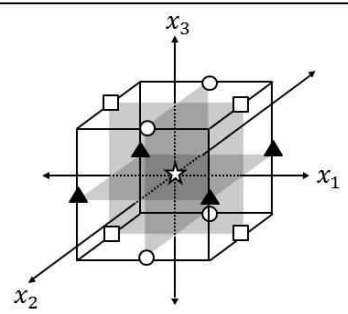

(b)
Fig. 4. Response surface methodology: (a) Central composite design; (b) Box-Behnken design
Table 1. Design variables for reduction of transmission torque ripple

\begin{tabular}{c|c|c|c|c}
\hline \multicolumn{2}{c|}{ Level } & \multicolumn{3}{c}{ Design variable } \\
\cline { 3 - 5 } \multicolumn{2}{c|}{} & $\alpha(\mathrm{mm})$ & $\beta(\mathrm{deg})$ & $\gamma(\mathrm{mm})$ \\
\hline Low & -1 & 28 & 1 & 3 \\
Central & 0 & 29.5 & 2.5 & 5.5 \\
High & +1 & 31 & 4 & 8 \\
\hline
\end{tabular}

Table 2. Box-Behnken design

\begin{tabular}{ccccccc}
\hline \multirow{2}{*}{$\begin{array}{c}\text { Standard } \\
\text { Order }\end{array}$} & $\alpha$ & $\beta$ & $\gamma$ & $\alpha$ & $\beta$ & $\gamma$ \\
\cline { 2 - 7 } & $x_{1}$ & $x_{2}$ & $x_{3}$ & $\mathrm{~mm}$ & $\operatorname{deg}$ & $\mathrm{mm}$ \\
\hline 1 & -1 & -1 & 0 & 28 & 1 & 5.5 \\
2 & +1 & -1 & 0 & 31 & 1 & 5.5 \\
3 & -1 & +1 & 0 & 28 & 4 & 5.5 \\
4 & +1 & +1 & 0 & 31 & 4 & 5.5 \\
5 & -1 & 0 & -1 & 28 & 2.5 & 3 \\
6 & +1 & 0 & -1 & 31 & 2.5 & 3 \\
7 & -1 & 0 & +1 & 28 & 2.5 & 8 \\
8 & +1 & 0 & +1 & 31 & 2.5 & 8 \\
9 & 0 & -1 & -1 & 29.5 & 1 & 3 \\
10 & 0 & +1 & -1 & 29.5 & 4 & 3 \\
11 & 0 & -1 & +1 & 29.5 & 1 & 8 \\
12 & 0 & +1 & +1 & 29.5 & 4 & 8 \\
13 & 0 & 0 & 0 & 29.5 & 2.5 & 5.5 \\
\hline
\end{tabular}

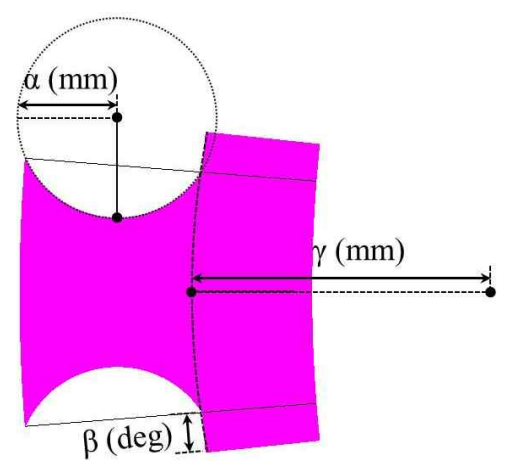

Fig. 5. Design variables of low-speed rotor

design variables, a has a value of \pm 1.682 , which has an experiment point 1.682 times larger than the maximum value of the preset design variable and 1.682 times smaller than the minimum value [7-8]. In this case, the range of the preset design variable becomes larger, and the shape of the proposed low-speed rotor may not be formed, or design variables that cannot be designed are generated. This results in difficulties in determining the range of the initial design variables. Thus, in this paper, the shape design of the MGM was performed using BBD. The design variables for reduction of the transmission torque ripple of the MGM are shown in Table 1 and Fig. 5. The experiment, based on BBD, was set up as shown in Table 2.

\subsection{Polynomial regression analysis}

Polynomial regression analysis includes second or third order term for dependent variables, and interaction term 
Table 3. ANOVA Results of transmission torque

\begin{tabular}{c|ccccc}
\hline Source & $\mathrm{DF}$ & $\mathrm{SS}^{1)}$ & $\mathrm{MS}^{2)}$ & $\mathrm{F}^{3)}$ & $\mathrm{P}^{4)}$ \\
\hline Regression & 3 & 0.89 & 0.30 & 1.85 & 0.180 \\
\hline Linear & 3 & 0.89 & 0.30 & 1.85 & 0.180 \\
$\alpha$ & 1 & 0.03 & 0.03 & 0.22 & 0.657 \\
$\beta$ & 1 & 0.56 & 0.53 & 3.48 & 0.078 \\
$\gamma$ & 1 & 0.31 & 0.35 & 1.98 & 0.172 \\
\hline Residual Error & 11 & 1.73 & 0.14 & & \\
\hline Total & 14 & 2.58 & & & \\
\hline
\end{tabular}

Table 4. ANOVA Results of transmission torque ripple

\begin{tabular}{|c|c|c|c|c|c|}
\hline Source & DF & $\mathrm{SS}$ & MS & $\mathrm{F}$ & $\mathrm{P}$ \\
\hline Regression & 6 & 5671.34 & 978.16 & 105.55 & $<0.001$ \\
\hline Linear & 3 & 603.11 & 202.31 & 21.45 & $<0.001$ \\
\hline$\alpha$ & 1 & 29.22 & 28.15 & 3.34 & 0.093 \\
\hline$\beta$ & 1 & 551.64 & 551.64 & 58.78 & $<0.001$ \\
\hline$\gamma$ & 1 & 4.35 & 4.35 & 0.43 & 0.483 \\
\hline Square & 2 & 5012.02 & 2512.32 & 264.66 & $<0.001$ \\
\hline$\beta \beta$ & 1 & 4645.35 & 4645.35 & 533.12 & $<0.001$ \\
\hline$\gamma \gamma$ & 1 & 145.14 & 145.14 & 14.21 & 0.004 \\
\hline Interaction & 1 & 204.43 & 204.43 & 21.43 & 0.001 \\
\hline$\alpha \beta$ & 1 & 204.43 & 204.43 & 21.43 & 0.001 \\
\hline Residual Error & 8 & 68.45 & 8.23 & & \\
\hline Total & 14 & 5532.21 & & & \\
\hline \multicolumn{6}{|c|}{ 1) $S S_{\text {Regression }}=\sum(\hat{y}-\bar{y})^{2}, \quad S S_{\text {Error }}=\sum(y-\bar{y})^{2}$} \\
\hline \multicolumn{6}{|c|}{ 2) $M S_{\text {Regression }}=S S_{\text {Regression }} / k, M S_{\text {Error }}=S S_{\text {Error }} / n-k-1$} \\
\hline \multicolumn{6}{|c|}{ 3) $F=M S_{\text {Regression }} / M S_{\text {Error }}$} \\
\hline \multicolumn{6}{|c|}{ 4) $P$-value $<0.05$} \\
\hline
\end{tabular}

between dependent variables. In order to design the experiment using the BBD and determine whether each factor is a significant term using the ANOVA, an f-test is used. In Tables 3 to 4 , degree of freedom (DF) represents the number of points that can be freely changed under the given conditions; SSR is sum of square for regression; SSE is sum of square for residuals; and MS is mean square. Fvalue represents the test statistic for f-verification, which is used to verify whether the regression equation is significant in explaining the property of data. Where, $y$ is result of the experiment, $\bar{y}$ is average of the $y$ values and $\hat{y}$ is predicted value by the regression equation. In addition, p-value is the probability of significance.

When the p-value derived from this test is smaller than 0.05 , the corresponding factor is considered to be significant. However, if the $\mathrm{p}$-value is greater than 0.05 , the corresponding factor is not significant. Accordingly, this factor is not considered to be one that affects dependent variables. Therefore, when performing an ANOVA, it is necessary to remove the factors of which $p$-value are greater than 0.05 (i.e., the factors that are not significant) sequentially from larger values [7-14].

Through this process, factors of the terms of interaction and factors of the terms of squares whose p-values are greater than 0.05 were removed. Analysis was performed according to the BBD, and the results were used to perform a regression analysis of design variables $\alpha, \beta$, and $\gamma$ of the low-speed rotor. As a result of the variance analysis of the transmission torque, all square terms and interaction terms were found to be insignificant and were removed. For the transmission torque ripple, $\alpha \alpha$ of the square term and $\beta \gamma, \alpha \gamma$ of the interaction term were found to be insignificant and were removed. The ANOVA results of the transmission torque and the ripple are shown in Table 3 and 4 , respectively.

In Table 3, $\alpha$ and $\beta, \gamma$ in the linear term have a $\mathrm{p}$ value of 0.05 or greater and thus, they are not significant. However, because the linear term itself is significant, it is acceptable to perform regression analysis without removing these terms.

Thus, the transmission torque ripple can be estimated with a secondary model. However, for the transmission torque, only the main effect works, and therefore, the transmission torque can be estimated with a linear model. Regression analysis equations for each design variable are as follows:

$$
\begin{aligned}
f_{T}(\alpha, \beta, \gamma)= & 6.12-0.0443 \alpha-0.163 \beta+0.09 \gamma \\
f_{T R}(\alpha, \beta, \gamma)= & 344.2-6.66 \alpha-176.3 \beta+10.24 \gamma \\
& +15.865 \beta \beta-0.987 \gamma \gamma+3.143 \alpha \beta
\end{aligned}
$$

where $f_{T}(\alpha, \beta, \gamma)$ and $f_{T R}(\alpha, \beta, \gamma)$ are the estimation functions of the transmission torque and the transmission torque ripple, respectively.

\subsection{Design results and numerical analysis}

Considering the transmission torque and transmission torque ripple characteristic of the MGM, the multiobjective design optimization model of a low-speed model can be defined as

$$
\begin{array}{ll}
\text { Maximize } & f_{T}(\alpha, \beta, \gamma) \\
\text { Minimize : } & f_{T R}(\alpha, \beta, \gamma)
\end{array}
$$

The overlaid contour plot for design variables of the proposed low-speed rotor shape is shown in Fig. 6. In

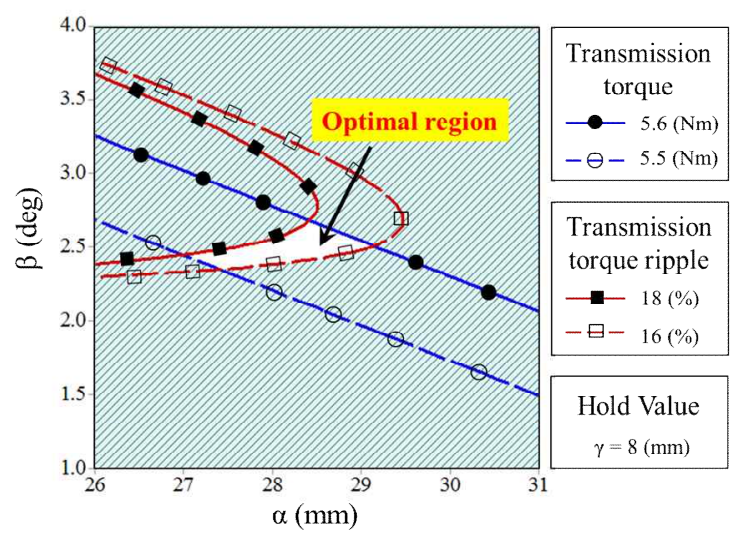

Fig. 6. Overlaid contour plot 
general, the overlaid contour plot has one design variable on the $\mathrm{x}$-axis and the other on $\mathrm{y}$-axis. If there are three or more variables, design variable should be fixed to a certain level. In the overlaid contour plot, the contour plot for each response is overlaid on one graph, and each contour plot represents the boundary of the response function value defined in the legend. The contour plot drawn with a solid line represents the lower limit, and the contour plot drawn with a dotted line represents the upper limit. The white region is the optimal region that satisfies all ranges indicated in the legend. Therefore, through an analysis of the response surface and the overlaid contour plot, the

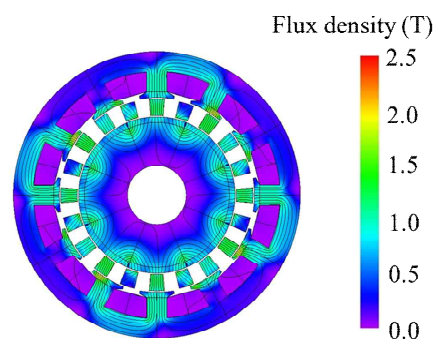

(a)

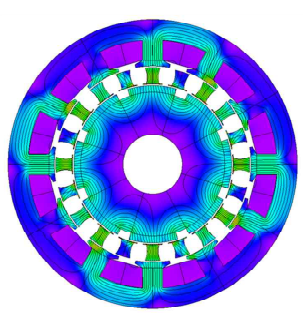

(b)
Fig. 7. Magnetic flux density contour: (a) Initial model; (b) Optimized model

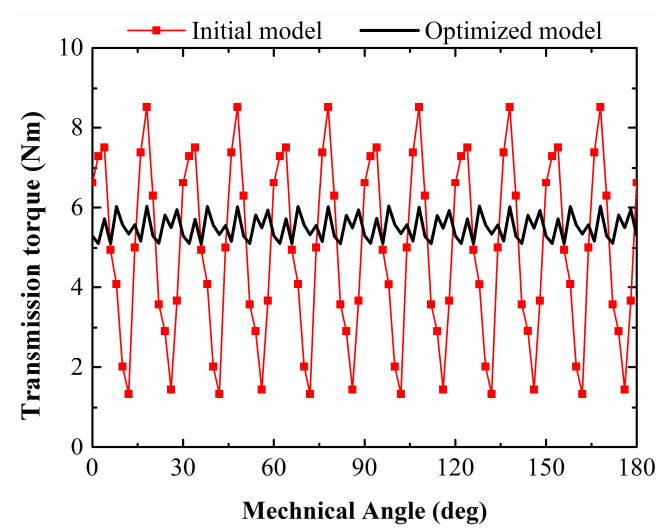

(a)

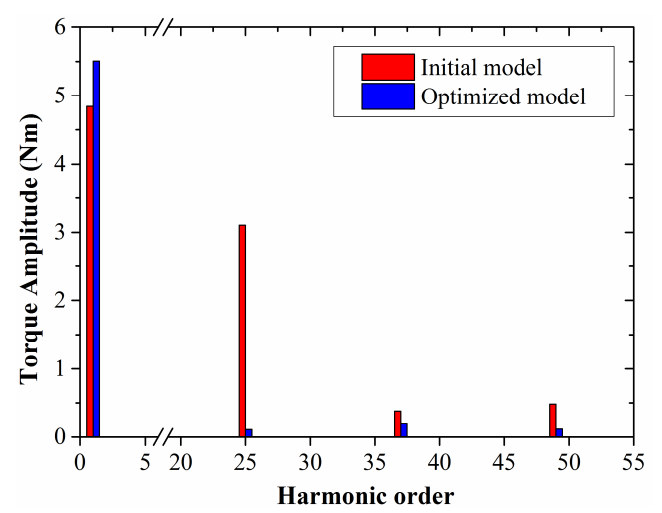

(b)

Fig. 8. Transmission torque: (a) Transmission torque waveforms; (b) Transmission torque harmonics optimal design variable $\alpha$ for the proposed low-speed rotor was determined to be $28 \mathrm{~mm}$. In addition, $\beta$ was $2.4^{\circ}$, and $\gamma$ was $8 \mathrm{~mm}$. Fig. 7 shows the magnetic flux density of the initial and optimized models. Fig. 8 shows the transmission torque waveforms of the initial and optimized models. As shown in Fig. 8(a), after optimization, the transmission torque ripple was reduced by $130.8 \%$ from that of the initial model. In addition, as shown in Fig. 8(b), the 25th, 37th, 49th harmonic components are dominant. In fact, the 25 th harmonic component is the main cause of for higher torque ripples in the MGM. Thus, it is mandatory to discard the specific 25 th torque harmonic component as much as possible. Remarkably, it is shown that the proposed design method is very effective in compensating for the targeted 25th torque harmonic component.

\section{Conclusion}

In this paper, an effective shape to reduce transmission torque ripple of the $\mathrm{MGM}$ is derived from a BBD. An analysis is conducted between independent variables selected by $\mathrm{BBD}$ and reaction variables using the nonlinear FEM based on 2-D numerical analysis. In addtion, the regression equation of the reaction variables is derived according to the independent variable by multiple regression analysis and an ANOVA. The validity of the optimized design is demostrated by comparing characteristics of the optimized model (derived from response surface analysis) and the initial model. As a result, the transmission torque ripple was reduced by $130.8 \%$ from that of the initial model.

\section{Acknowledgements}

This research was supported by Basic Science Research Program through the National Research Foundation of Korea (NRF) funded by the Ministry of Education (2015R1D1A3A01017536) and the Korea Institute of Energy Technology Evaluation and Planning(KETEP) and the Ministry of Trade, Industry \& Energy (MOTIE) of the Republic of Korea (No. 20164010201020).

\section{References}

[1] K. Atallah and D. Howe, "A Novel HighPerformance Magnetic Gear," IEEE Trans. Magn., vol. 37, no. 4, pp. 2844-2846, 2001.

[2] Peter Omand Rasmussen, Torben Ole Andersen, Frank T. Jørgensen, and Orla Nielsen, "Development of a High-Performance Magnetic Gear," IEEE Trans. Ind. Appl., vol. 41, no. 3, pp. 764-770, 2005.

[3] Noboru Niguchi and Katsuhiro Hirata, "Torque- 
Speed Characteristics Analysis of a Magnetic-Geared Motor Using Finite Element Method Coupled With Vector Control," IEEE Trans. Magn., vol. 49, no. 5, pp. 2401-2404, 2013.

[4] K. T Chau, Dong Zhang, J, Z. Jiang, Chunhua Liu, and Yuejin Zhang, "Design of a Magnetic-Geared Outer-Rotor Permanent-Magnet Brushless Motor for Electric Vehicles," IEEE Trans. Magn., vol. 43, no. 6, pp. 2504-2506, 2007.

[5] L. Jian, K. T. Chau and J. Z. Jiang, "A magneticgeared outer-rotor permanent-magnet brushless machine for wind power generation," IEEE Trans. Ind. Appl., vol. 45, no. 3, pp. 954-962, 2009.

[6] H. M. Shin, J. H. Chang, "Design of Coaxial Magnetic Gear for Improvement of Torque Characteristics," J. Magn., vol. 19, no. 4, 2014.

[7] B. H. Lee, J. P. Hong and J. H. Lee, "Optimum Design Criteria for Maximum Torque and Efficiency of a Line-Start Permanent-Magnet Motor using Response Surface Methodology and Finite Element Method," IEEE Trans. Magn., vol. 48, no. 2, 2012.

[8] H. M. Hasanien, A. S. Abd-Rabou and S. M. Sakr, "Design optimization of transverse flux linear motor for weight reduction and performance improvement using response surface methodology and genetic algorithms," IEEE Trans. Energy Convers., vol. 25, no. 3, pp. 598-605, 2010.

[9] S. J. Kim, C. H. Kim, S. Y. Jung, Y. J. Kim, “Optimal Design of Novel Pole Piece for Power Density Improvement of Magnetic Gear Using Polynomial Regression Analysis," IEEE Trans. Energy Convers., vol. 30, no. 3, pp. 1171-1179, 2015.

[10] S. J. Kim, C. H. Kim, S. Y. Jung, Y. J. Kim, “Shape Optimization of a Hybrid Magnetic Torque Converter Using the Multiple Linear Regression Analysis," IEEE Trans. Magn., vol. 52, no. 3, pp. 8102504, 2016.

[11] S. H. Lee, Y. J. Kim, K. S. Lee, S. J. Kim, "Multiobjective Optimization Design of Small-Scale Wind Power Generator with Outer Rotor Based on BoxBehnken Design," IEEE Trans. Appl. Super-cond., vol. 26, no. 4, pp.5202605, 2016.

[12] N. W. Frank, S. Pakdelian, H. A. Toliyat, "Passive Suppression of Transient Oscillations in the Concentric Planetary Magnetic Gear," IEEE Trans. Energy Convers., vol. 26, no. 3, pp. 933-939, 2011.

[13] M. H. Jeon, D. H. Kim and C. E. Kim, "Optimum Design of BLDC Motor for Cogging Torque Minimization Using Genetic Algorithm and Response Surface Method," J. Elect. Eng. Technol., vol. 1, no. 4, pp. 466-471, 2006.

[14] D. K. Woo, I. W. Kim and H. K. Jung, "Optimal Rotor Structure Design of Interior Permanent Magnet Synchronous Machine based on Efficient Genetic Algorithm Using Kriging Model," J. Elect. Eng. Technol., vol. 7, no. 4, pp. 530-537, 2012.

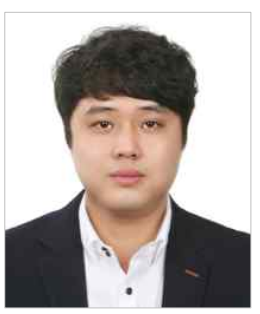

Sung-Jin Kim He received the B.S and M.S degrees in electrical engineering from Chosun University, Gwangju, Korea, in 2011 and 2013, respectively. He is currently pursuing a Ph.D degree in electrical engineering at the same University. His research interests are numerical analysis and design of linear and PM machineries.

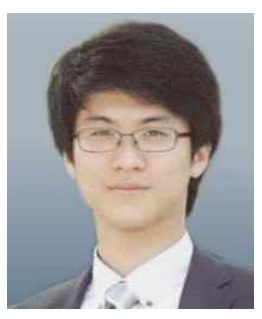

Eui-Jong Park He received B.S and M.S degree in department of electrical engineering from Chosun University, Gwang-ju, Korea in 2013 and 2015, respectively. Since 2015, he is doing a $\mathrm{Ph} . \mathrm{D}$ course in department of electrical engineering from Chosun University, Gwang-ju, Korea. His research interests are numerical analysis and design of linear machineries and PM machineries.

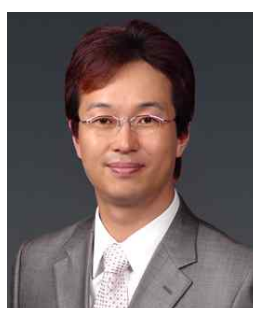

Yong-Jae Kim He received the B.S degree in electrical engineering from Chosun University, Gwangju, Korea, in 1996, and the M.S and Ph.D degrees in electrical engineering from Musashi Institute of Technology, Tokyo, Japan, in 2003 and 2006, respectively. From 2006 to 2007, he was a Researcher of electrical and electronic engineering with the Musashi Institute of Technology, Tokyo, Japan. He is currently an Associate Professor with the Department of Electrical Engineering, Chosun University, Gwangju, Korea. His current research interests include the design and analysis of electric machines. 